\title{
AMP-Activated Protein Kinase: Its Regulation by Different Sites
}

\author{
Katherine M Allen and Asish K Saha* \\ Department of Medicine, Boston University Medical Center, USA
}

Submission: June 21, 2017; Published: June 30, 2017

*Corresponding author: Asish K Saha, Department of Medicine, Boston University Medical Center, USA. Tel: 617-638-7169; Fax: 617-638-7094; Email: aksaha@bu.edu.

\begin{abstract}
Type 2 diabetes (T2D), as well as other metabolic diseases, is an increasing global health concern and many of the mechanisms of both the disease and its current drug treatments have not been fully described. Numerous pharmaco 7 logical agents, natural compounds, and hormones are known to activate AMP-activated protein kinase (AMPK), either directly or indirectly-some of which (for example, metformin and thiazolidinediones) are currently used to treat T2D. It has been shown that the anti-diabetic class of drugs, the thiazolidinediones, works via both a known PPAR $\gamma$-dependent, and a lesser known PPAR $\gamma$-independent mechanism of action. This PPAR $\gamma$-independent mechanism likely involves

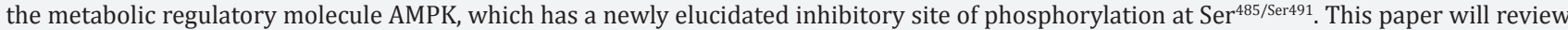
the regulation of the AMPK pathway by both its activation and inhibitory sites and the potential for future improvements in targeting AMPK for the treatment of T2D.
\end{abstract}

\section{AMPK Regulation}

AMP-activated protein kinase (AMPK) is a molecule found in almost every cell in the human body. The molecule is a serine/threonine kinase consisting of three subunits, a catalytic $\alpha$-subunit and regulatory $\beta$ - and $\gamma$-subunits, each of which has multiple isoforms [1-3]. Different isoforms tend to be found in different tissues, the most common example of which is $\alpha 1$ containing isoforms which are most predominant in the liver and adipose tissue, and $\alpha 2$-containing isoforms which are most predominant in skeletal muscle, brain, and heart [4]. AMPK's primary purpose is regulation of cellular metabolic functions including, but not limited to, glucose and lipid homeostasis, adipokine driven regulation of food intake, and body weight [5]. When activated, AMPK phosphorylates many downstream targets that lead to the inhibition of pathways that consume energy, such as fatty acid synthesis, cholesterol synthesis, and gluconeogenesis [6]. This downstream phosphorylation also leads to the stimulation of pathways that generate energy, glucose uptake, fatty acid oxidation, glycolysis, and food intake to name a few [6]. Activation of AMPK requires both an increase in the intracellular AMP: ATP ratio and phosphorylation of Thr172 on the "activation loop" [7] of the $\alpha$-subunit by one of its three upstream kinases: the tumor-suppressor liver kinase B1 (LKB1)
$[8,9]$, the calcium-dependent calcium/calmodulin-dependent protein kinase kinase $\beta$ (СаMKK $\beta$ ) [10], or transforming growth factor- $\beta$ activated protein kinase-1 (TAK1) [11]. Aside from these three kinases, there are a vast number of known, upstream AMPK activators of physiological, hormonal, natural, and pharmacological origin [6]. Physiological activators of AMPK are thought to be exercise and calorie restriction as they help increase the cellular ratio of AMP: ATP [12]. Resveratrol, a polyphenol found in red wine, rooibos, a South American plant, berberine, an alkaloid found in certain plants, and $\alpha$-lipoic acid, a short-chain fatty acid, are just a few of the naturally occurring compounds thought to activate AMPK as well as the hormones leptin, adiponectin, and interleukin-6 (IL-6) [13]. Pharmacological activators of AMPK include 5-Aminoimidazole4-carboxamide riboside (AICAR), biguanides such as Metformin and the thiazolidinediones which include troglitazone, rosiglitazone, and pioglitazone [6]. Although Thr172 on the $\alpha$-subunit is regarded as the main phosphorylation/activation site, changes in AMPK activity have recently been observed in the absence of altered Thr172 phosphorylation.

\section{AMPK inhibitory site}

AMPK activity may be impacted by one of several other phosophorylation sites on AMPK with less defined functions 
$[14,15]$. One such site is $\operatorname{Ser}^{485 / 491}$ on the $\alpha 1 / \alpha 2$ subunit, which will be discussed below in $\mathrm{f}$ AMPK Ser ${ }^{485 / 491}$. As previously mentioned, recent data suggests that the Ser ${ }^{485 / 491}$ site on AMPK's $\alpha 1 / \alpha 2$ subunit may play an important role in modulating AMPK activity. Phosphorylation of this site has been shown to inhibit AMPK in several tissues, though its role in muscle or liver has not been investigated. $\operatorname{Ser}^{485}$ on the $\alpha 1$ subunit has been shown to be phosphorylated by Akt $[16,17]$, protein kinase A (PKA) [18-20], or autophosphorylation [18] in various cell types and tissues, such as heart [16], adipocyte [15, 18], and vascular smooth muscle cells [17]. Similarly, Ser ${ }^{491}$ of the $\alpha 2$ subunit can be phosphorylated by PKA $[18,19]$, p70S6K (19), or autophosphorylation in tissues such as adipocytes, hypothalamus [21], heart [16], and HEK293 cells [18], resulting in reduced AMPK activity. Although previous studies suggested that $\operatorname{Ser}^{491}$ is also an Akt phosphorylation site, a recent study by Hawley et al. showed that Akt does not phosphorylate Ser ${ }^{491}$ in a cell-free assay [22]. In heart, it has been shown that insulin and IGF-1 rapidly stimulate phosphorylation of this site through Akt, leading to a decrease in AMPK activity. In the hypothalamus, Dagon et al. [21] showed that p70S6K phosphorylates $\alpha 2 \mathrm{AMPK}$ $\mathrm{Ser}^{491}$ to inhibit AMPK and decrease food intake. Some of these changes in serine phosphorylation correlate inversely with that of $\mathrm{Thr}^{172}$; however, in many instances, there seems to be discordance between the two phosphorylation sites.

Whether Ser ${ }^{485 / 491}$ phosphorylation plays a role in the inhibition of AMPK seen in diabetic models is unknown. Notably, recent studies suggest that phosphorylation of this site may be increased under conditions of excess nutrients. For example, it was shown that when the MIN6 cell line of pancreatic $\alpha$-cells were switched from low glucose $(3 \mathrm{mM})$ culture medium to high glucose $(25 \mathrm{mM})$ for either $1 \mathrm{~h}$ or overnight, phosphorylation of $\alpha 1$ AMPK Ser ${ }^{485}$ was increased [23]. In this experiment, phosphorylation at this site was inversely correlated to phosphorylation of $\alpha$ AMPK $\mathrm{Thr}^{172}$.

A better understanding of how AMPK is regulated at a molecular level is important for improving drug-targeting strategies for T2D [24]. Phosphorylation of AMPK at $\mathrm{Thr}^{172}$ of the activation loop is essential for the activation of AMPK and is often used as readout for activity. However, our data adds to a growing number of studies showing that phosphorylation of AMPK at $\operatorname{Ser}^{485 / 491}$ can inhibit AMPK activity, even in the absence of changes in p-AMPK Thr ${ }^{172}$ [25]. Although p-AMPK Thr ${ }^{172}$ is often used as a measure of AMPK activity, these data suggest that phosphorylation of AMPK at $\operatorname{Ser}^{485 / 491}$ can inhibit AMPK activity independent of changes in $\mathrm{p}$-AMPK Thr ${ }^{172}$ and that inhibition of p-AMPK Thr ${ }^{172}$ is not required for Ser ${ }^{485 / 491}$ phosphorylation to induce inhibition of AMPK (Figure 1) activity. We showed, for the first time, that PKD1 activation can stimulate phosphorylation of AMPK Ser ${ }^{485 / 491}$ in skeletal muscle cells, resulting in impaired insulin signaling [26]. Not only does this work identify a novel kinase that can inhibit AMPK by serine phosphorylation at this site, but it also presents a possible mechanism by which AMPK may be inhibited by excess nutrients since DAG (which activates PKD) is known to be activated by high glucose and lipids.

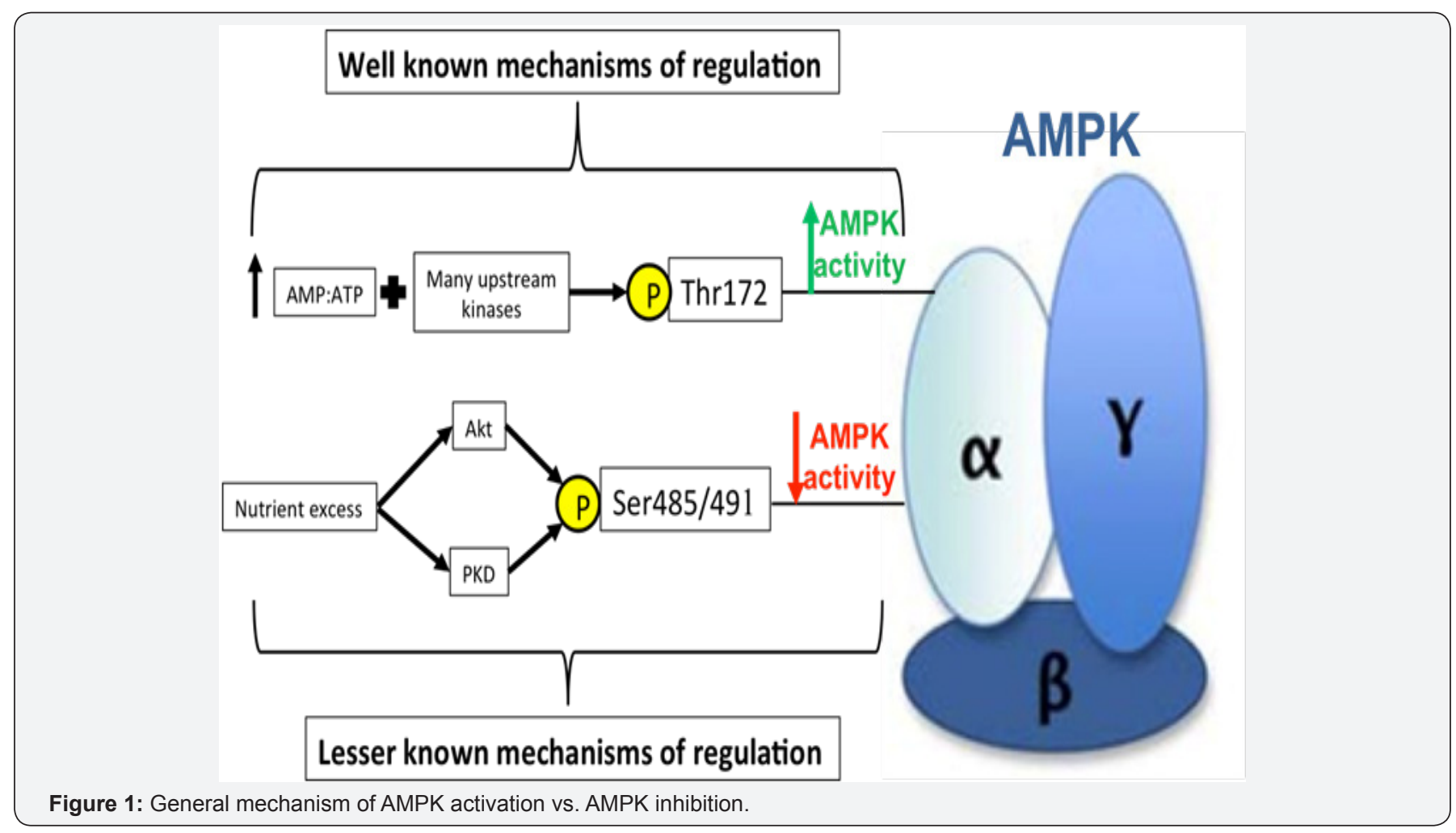




\section{Conclusion}

In a very recent study we have found that the TZD, troglitazone, activated AMPK via phosphorylation of $\mathrm{Thr}^{172}$ in cultured hepatocytes [27]. These data support the idea of a PPAR $\gamma$-independent mechanism of TZD action that has been previously proposed. It was also shown that troglitazone phosphorylated AMPK at its Ser485inhibitory site. To our knowledge, this is the first time we have seen AMPK increasingly phosphorylated at both $\mathrm{Thr}^{172}$ and $\mathrm{Ser}^{485}$. This leaves the possibility for new research to examine further into both the mechanisms of Ser ${ }^{485}$ phosphorylation and the effects this has on the AMPK molecule as a whole. A better understanding of AMPK regulation and the factors contributing to its inhibition could help identify novel AMPK activators, as well as other molecules that could be targeted to AMPK. It is well known that both a rise in the AMP: ATP ratio along with phosphorylation at AMPK Thr $^{172}$ increases AMPK activity. A lesser known mechanism of regulation involves phosphorylation of AMPK at Ser ${ }^{485 / 491}$ which leads to decreased AMPK activity. Cellular conditions caused by excess nutrients, such as high insulin and high glucose (common in type 2 diabetes) have been shown to increase both Akt and PKD which directly phosphorylate AMPK at $\operatorname{Ser}^{485 / 491}[25,26]$.

\section{References}

1. Grunberger G, Abelseth JM, Bailey TS, Bode BW, Handelsman Y, et al. (2014) Consensus statement by the American Association of Clinical Endocrinologists/American College of Endocrinology Insulin Pump Management Task Force. Endocr Pract 20(5): 463-489.

2. Davidson PC, Hebblewhite HR, Steed RD, Bode BW (2008) Analysis of guidelines for basal-bolus insulin dosing: basal insulin, correction factor, and carbohydrate-to-insulin ratio. Endocr Pract 14(9): 10951101.

3. King AB, Armstrong DU (2007) A prospective evaluation of insulin dosing recommendations in patients with type 1 diabetes at near normal glucose control: Basal dosing. J Diabetes Sci Technol 1(1): 3641.

4. King AB, Armstrong DU (2007) A prospective evaluation of insulin dosing recommendations in patients with type 1 diabetes at near normal glucose control: Bolus Dosing. J Diabetes Sci Technol 1(1): 4246.

5. Kuroda A, Yasuda T, Takahara M, Sakamoto F, Kasami R, et al. (2012) Carbohydrate-to-insulin ratio is estimated from 300-400 divided by total daily insulin dose in type 1 diabetes patients who use the insulin pump. Diabetes Technol Ther 14(11): 1077-1080.

6. Alcantara-Aragon V, Gonzalez C, Corcoy R, Ubeda J, Chico A (2015) Carbohydrate-to-Insulin Ratio in a Mediterranean Population of Type 1 Diabetic Patients on Continuous Subcutaneous Insulin Infusion Therapy. J Diabetes Sci Technol 9(3): 588-592.

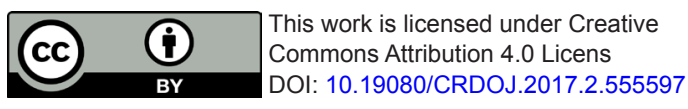

